\title{
A CHARACTERIZATION OF SPECTRAL OPERATORS
}

\author{
MEHDI RADJABALIPOUR
}

(Communicated by Palle E. T. Jorgensen)

\begin{abstract}
A characterization of spectral operators due to N. Dunford is simplified. Especially, his complicated Condition (D) is replaced by a very simple one.
\end{abstract}

Dunford [2;3; p. 2147] showed that an operator $T$ defined on a weakly complete Banach space $Y$ is spectral if and only if it satisfies the following conditions (i)-(iv).

(i) Dunford's analyticity condition (A). $T$ has the single-valued extension property; that is, for every $x \in Y$ there exists a smallest closed set $\sigma_{T}(x)$, called the local spectrum of $T$ at $x$, and a unique analytic function $f: \mathbf{C} \backslash \sigma_{T}(x) \rightarrow Y$, called the local resolvent of $T$ at $x$, such that $(z-T) f(z) \equiv x$.

(ii) Dunford's boundedness condition (B). There exists a bound $K$ such that $\|x\| \leq K\|x+y\|$ whenever $\sigma_{T}(x) \cap \sigma_{T}(y)=\varnothing$.

(iii) Dunford's closure condition (C). For every closed set $F \subset \mathbf{C}$, the set $Y_{T}(F):=\left\{x: \sigma_{T}(x) \subset F\right\}$ is closed .

(iv) Dunford's decomposability condition (D). Let $\mathscr{S}_{1}(T)$ denote the collection of all $\delta \subset \mathbf{C}$ for which $Y_{T}(\delta)+Y_{T}(\mathbf{C} \backslash \delta)$ is dense in $Y$. Assuming $T$ satisfies condition (B), it follows that $Y$ is the direct sum of $\overline{Y_{T}(\delta)}$ and $\overline{Y_{T}(\mathbf{C} \backslash \delta)}$ and the corresponding projections $E(\delta)$ and $E(\mathbf{C} \backslash \delta)$ are bounded by $K$. Let $\mathscr{S}_{2}(T)$ denote the collection of all $\delta \in \mathscr{S}_{1}(T)$ such that for every $\varepsilon>0$ there exist $x_{1}, x_{2} \in Y$ with $\left\|x_{1}+x_{2}-x\right\|<\varepsilon, \sigma_{T}\left(x_{1}\right) \subset$ $\delta \cap \sigma_{T}(x)$, and $\sigma_{T}\left(x_{2}\right) \subset \sigma_{T}(x) \backslash \delta$. We assume that for every $\lambda \in \mathbf{C}$ and $r>0$ there exist $\delta, \mu_{n}, \gamma_{n} \in \mathscr{S}_{2}(T)(n=1,2, \ldots)$ such that $\lambda \in \delta^{0}$, $\operatorname{diam}(\delta) \leq r, \mu_{n} \subset \delta, \gamma_{n} \subset \mathbf{C} \backslash \delta, \mu_{n}$ closed, $\gamma_{n}$ closed $(n=1,2, \ldots)$, and $x=\lim \left[E\left(\mu_{n}\right) x+E\left(\gamma_{n}\right) x\right]$.

The purpose of this paper is to simplify the above criterion. For an arbitrary operator $T$, and a subset $\delta$ of $\mathbf{C}$, we define $S_{T}(\delta)$ to be the set of all $x \in Y$ such that $(z-T) f(z) \equiv x$ for some analytic $Y$-valued function $f$ defined on an open set containing $\mathbf{C} \backslash \delta$.

Received by the editors March 20, 1990.

1980 Mathematics Subject Classification (1985 Revision). Primary 47B40.

Key words and phrases. Spectral operator, single-valued extension property, Dunford's conditions (B), (C), and (D). 
Theorem. $A$ (bounded linear) operator $T$ on a weakly complete Banach space $Y$ is spectral if and only if $T$ satisfies the following conditions.

(a) There exists $K>0$ such that $\|x\| \leq K\|x+y\|$ whenever $x \in S_{T}(\delta)$, $y \in S_{T}(\mathbf{C} \backslash \delta)$, and $\delta=\bar{\delta} \subset \mathbf{C}$.

(b) $S_{T}(\Delta)$ is closed and $S_{T}(\Delta)+S_{T}(\mathbf{C} \backslash \Delta)$ is dense in $Y$ whenever $\Delta$ is a closed (vertical or horizontal) half plane.

Proof. We first prove the sufficiency of the conditions. Assume the equation $(z-T) f(z) \equiv 0$ has a nonzero analytic solution $f$. Let $N(\lambda ; r)$ be an arbitrary open disc lying entirely in the domain of $f$. Since $(z-T)(z-\lambda)^{-1} f(\lambda) \equiv f(\lambda)$ for $z \neq \lambda$ it follows that $f(\lambda) \in S_{T}(\{\lambda\})$. Define $g(z)=(z-\lambda)^{-1}[f(z)-f(\lambda)]$ for $z \in N(\lambda ; r) \backslash\{\lambda\}$, and $g(\lambda)=f^{\prime}(\lambda)[1$, p. 2]. Then $(z-T) g(z) \equiv-f(\lambda)$ for $z \in N(\lambda ; r)$ and thus $-f(\lambda) \in S_{T}(\mathbf{C} \backslash N(\lambda ; r))$. In view of condition (B), $\|f(\lambda)\| \leq K\|f(\lambda)-f(\lambda)\|=0$. Since $\lambda$ is arbitrary, $f \equiv 0$. Thus $T$ satisfies condition (A) and $S_{T}\left(\cap F_{\alpha}\right)=\bigcap S_{T}\left(F_{\alpha}\right)$ for any family $\left\{F_{\alpha}\right\}$ of subsets of $\mathbf{C}$. Also in view of (a), $T$ satisfies condition (B).

Now, let $\delta \subset \mathbf{C}$ be such that $Y=\bar{S}_{T}(\delta) \oplus \bar{S}_{T}(\mathbf{C} \backslash \delta)$. Define $E(\delta)$ to be the projection onto $\bar{S}_{T}(\delta)$ that is parallel to $\bar{S}_{T}(\mathbf{C} \backslash \delta)$. Because $(z-T) E(\delta) f(z) \equiv E(\delta) x$ whenever $f$ is the local resolvent of $T$ at $x$, it follows that $\sigma_{T}(E(\delta) x) \subset \sigma_{T}(x) \cap \bar{\delta}$ provided that $S_{T}(\bar{\delta})$ is closed. Moreover, $\|E(\delta)\| \leq K, E\left(\delta_{1}\right) E\left(\delta_{2}\right)=E\left(\delta_{1}\right)$ if $\delta_{1} \subset \delta_{2}$, and $E\left(\delta_{1}\right) E\left(\delta_{2}\right)=0$ if $\delta_{1} \cap \delta_{2}=\varnothing$. In particular, $E(\Delta)$ and $E(\mathbf{C} \backslash \Delta)$ are defined for any closed (vertical or horizontal) half plane $\Delta$.

We claim $E(R)$ can be defined for any semiclosed rectangle $R$ of the form $(a, b] \times(c, d]$. Let $x \in Y$. Let $\Delta$ be as in (b) and assume $\left\{\Delta_{n}\right\}$ is a strictly decreasing sequence of closed half planes converging to $\Delta$. Because $u_{n}=E(\mathbf{C} \backslash \Delta) E\left(\Delta_{n}\right) x$ is bounded, every subsequence of $\left\{u_{n}\right\}$ has a weakly convergent subsequence. Let $u$ be the limit of any weakly convergent subsequence of $\left\{u_{n}\right\}$. Since $u_{n} \in S_{T}\left(\Delta_{N}\right) \cap \bar{S}_{T}(\mathbf{C} \backslash \Delta)$ for all $n \geq N$, it follows that $u \in S_{T}\left(\Delta_{N}\right) \cap \bar{S}_{T}(\mathbf{C} \backslash \Delta)$ and hence $u \in S_{T}(\Delta) \cap \bar{S}_{T}(\mathbf{C} \backslash \Delta)=\{0\}$. Thus $v_{n}=E(\mathbf{C} \backslash \Delta) E\left(\mathbf{C} \backslash \Delta_{n}\right) x$ converges weakly to $E(\mathbf{C} \backslash \Delta) x$. Therefore, a sequence $w_{n}$ of finite convex combinations of $v_{n}$ converges strongly to $E(\mathbf{C} \backslash \Delta) x$. Since $\sigma_{T}\left(v_{n}\right) \subset \sigma_{T}(x) \backslash \Delta, \sigma_{T}\left(w_{n}\right) \subset \sigma_{T}(x) \backslash \Delta$ for all $n$. Summing up, we have shown that for every $x \in Y, \Delta$ as in (b), and $\varepsilon>0$, there exists $w \in Y$ such that $\|E(\mathbf{C} \backslash \Delta) x-w\|<\varepsilon$ and $\sigma_{T}(w) \subset \sigma_{T}(x) \backslash \Delta$. Thus by consecutive applications of this result to the half planes $\{z: \operatorname{Re} z \leq a\},\{z: \operatorname{Re} z \leq b\}$, $\{z: \operatorname{Im} z \leq c\}$, and $\{z: \operatorname{Im} z \leq d\}$, we obtain vectors $x_{1}$ and $x_{2} \in Y$ such that $\sigma_{T}\left(x_{1}\right) \subset \sigma_{T}(x) \backslash R, \sigma_{T}\left(x_{2}\right) \subset S_{T}(R)$, and $\left\|x-x_{1}-x_{2}\right\|<\varepsilon$. Thus $S_{T}(R)+S_{T}(\mathbf{C} \backslash R)$ is dense in $Y$ and hence $Y=\bar{S}_{T}(R) \oplus \bar{S}_{T}(\mathbf{C} \backslash R)$.

Let $R_{0}=(-a, a] \times(-a, a]$ be a semiclosed square containing $\sigma(T)$. For each $n \in \mathbf{N}$, let $\left\{D_{n k}: k=1,2, \ldots, n\right\}$ be the family of disjoint semiclosed squares of diameter $a \sqrt{2} / 2^{n}$ covering $R_{0}$. It is clear that $E$ is a bounded additive set function on the Boolean algebra generated by $\left\{D_{n k}: k=\right.$ $\left.1,2, \ldots, 4^{n} ; n=1,2, \ldots\right\}$. Define $S_{n}=\sum_{k=1}^{4^{n}} z_{n k} E\left(D_{n k}\right)$, where $z_{n k}$ is 
the centre of $D_{n k}$. For $m>n$, we have

$$
\left\|S_{m}-S_{n}\right\|=\left\|\sum_{k=1}^{4^{n}} c_{m k} E\left(D_{m k}\right)\right\| \leq 4 a \sqrt{2} / 2^{n},
$$

where $c_{m k}$ is the difference between the centre of $D_{m k}$ and the centre of $D_{n j}$ such that $D_{m k} \subset D_{n j}\left[3 ;\right.$ p. 2181]. Hence $\left\{S_{n}\right\}$ converges to a scalar-type spectral operator $A$ [3; p. 2192]. Obviously, $A T=T A$. We claim $T-A$ is quasinilpotent. Let $T_{n k}$ and $A_{n k}$ be the restrictions of $T$ and $A$ to $E\left(D_{n k}\right) Y=$ $\bar{S}_{T}\left(D_{n k}\right)$ for all possible pairs $(n, k)$. Since $S_{T}\left(\bar{D}_{n k}\right)$ is the intersection of four spectral manifolds corresponding to closed half planes, it follows that $S_{T}\left(\bar{D}_{n k}\right)$ is closed and hence $\sigma\left(T \mid \bar{S}_{T}\left(D_{n k}\right)\right) \subset \sigma\left(T \mid S_{T}\left(\bar{D}_{n k}\right)\right) \subset \bar{D}_{n k}$ [1, p. 23]. Also, let $S_{m n k}=S_{m} \mid \bar{S}_{T}\left(D_{n k}\right)$ and observe that $\left\|\left(z-S_{m n k}\right)^{-1}\right\| \leq K / \operatorname{dist}\left(z, D_{n k}\right)$. Letting $m \rightarrow \infty$ it yields $\left\|\left(z-A_{n k}\right)^{-1}\right\| \leq K$ dist $/\left(z, D_{n k}\right)$ for all possible $(n, k)$. Thus $\sigma\left(A_{n k}\right) \subset \bar{D}_{n k}$ and hence $\sigma\left(T_{n k}-A_{n k}\right) \subset\left\{\alpha-\beta: \alpha \in \bar{D}_{n k}\right.$, $\left.\beta \in \bar{D}_{n k}\right\}$ [8, Chapter 0]. Therefore, $\sigma\left(T_{n k}-A_{n k}\right)$ lies in a disc of radius $a \sqrt{2} / 2^{n-1}$ for all possible $(n, k)$. It follows that

$$
\sigma(T-A) \subset \bigcup_{k=1}^{4^{n}} \sigma\left(T_{n k}-A_{n k}\right) \subset\left\{\lambda:|\lambda|<a \sqrt{2} / 2^{n-1}\right\}
$$

for all $n$, and thus $\sigma(T-A)=\{0\}$. Therefore, $T-A$ is quasinilpotent and consequently, $T$ is spectral.

The necessity of the conditions is an immediate consequence of Dunford's characterization.

Remarks. (1) We have shown that if $T$ satisfies condition (a) of the theorem, then it necessarily satisfies condition (A).

(2) A sort of strong regularity of $E$ depicted by $x=\lim \left[E\left(\mu_{n}\right)+E\left(\gamma_{n}\right)\right] x$ required in Dunford's condition (D) is not needed in our criterion. We at most require $S_{T}(\Delta)+S_{T}(\mathbf{C} \backslash \Delta)$ to be dense in $Y$. In other words, we need only the closed (vertical or horizontal) half planes to belong to $\mathscr{S}_{1}(T)$.

(3) Dunford's requirement that $S_{T}(F)$ be closed for every set $F$ is reduced in our criterion to the requirement that $S_{T}(\Delta)$ be closed for all closed half planes. The following example shows that $T$ may fail to be spectral if, in Condition (b) of the theorem, $\Delta$ is assumed only to be a closed right or upper half plane.

(4) Some of the ideas are taken from the proof of Theorem 1 of [7].

Example. Let $V$ be a nonunitary contraction operator on a Hilbert space $K$ with $\sigma(B)=\{1\}$ [5, Problem 150]. Let $\varphi$ be a conformal mapping from the unit disc onto $\Delta_{1}=\left\{\mathrm{re}^{i \theta}: 0 \leq r \leq 1,0 \leq \theta \leq \pi / 4\right\}$ such that $\varphi(1)=0$. Let $A=\varphi(V)$. Then $\sigma(A)=\{0\}$ and $\Delta_{1}$ is a spectral set for $A[4, \S 1.1$; 9, p. 143; 6, proof of Proposition 1]. Then the set $\Delta_{n}=\left\{\mathrm{re}^{i \theta}: 0 \leq r \leq 1\right.$, $0 \leq \theta \leq \pi / 4 n\}$ is a spectral set for $A^{1 / n}=\varphi_{n}(A)$, where $\varphi_{n}\left(\mathrm{re}^{i \theta}\right)=r^{1 / n} e^{i \theta / n}$ 
and $\mathrm{re}^{i \theta} \in \Delta_{n} \quad(n \in \mathbf{N})$. Let $T=A \oplus A^{1 / 2} \oplus \cdots$. As it is shown in the proof of Proposition 1 of [6], $\sigma(T)=[0,1], \bar{S}_{T}(\{0\})=H$, and $T$ satisfies the single-valued extension property where $H=K \oplus K \oplus \cdots$. Thus $S_{T}(\{0\})$ is not closed [1; p. 23]. Hence, in the light of Dunford's criterion, $T$ is not spectral. Let $0 \neq x \in H$ be arbitrary. We claim $0 \in \sigma_{T}(x)$. Let $f$ be the local resolvent of $T$ at $x$ and assume, if possible, that $0 \notin \sigma_{T}(x)$. Let $x_{n}$ be the projection of $x$ in the $n$th copy of $K$. Then $\sigma_{T}\left(x_{n}\right)=\sigma_{A^{1 / n}}\left(x_{n}\right) \subset \sigma_{T}(x)$. Since $A^{1 / n}$ is quasinilpotent, $x_{n}=0 \quad(n \in \mathbf{N})$. Thus $x=0$, a contradiction. Thus condition (a) holds trivially. Moreover, if $\delta$ is any Borel set, then $S_{T}(\delta)+S_{T}(\mathbf{C} \backslash \delta)$ is dense in $H$ because it contains $S_{T}(\{0\})$. Now, let $\Delta$ be any closed horizontal half plane. Then, either $[0,1] \subset \Delta$, in which case $S_{T}(\Delta) \supset S_{T}(\sigma(T))=H$, or $0 \notin \Delta$, from which follows $S_{T}(\Delta)=\{0\}$. Finally, assume $\Delta$ is a closed right half plane. Then $S_{T}(\Delta)=\{0\}$ if $0 \notin \Delta$, and $S_{T}(\Delta)=H$ if $0 \in \Delta$.

Added in proof. We are grateful to the referee who brought to our attention a preprint of Professor B. L. Wadhwa's talk [10], where he shows that $T$ is spectral if and only if $T$ satisfies the above Dunford's Conditions (A), (B), (C) and the following Condition $\left(\mathrm{D}^{\prime}\right): S_{T}(\delta)+S_{T}(\mathbf{C} \backslash \delta)$ is dense in $Y$ for all closed sets $\delta$. Thus the main result of our paper extends Wadhwa's result by omitting Condition $(\mathrm{A})$ and restricting Conditions $(\mathrm{C})$ and $\left(\mathrm{D}^{\prime}\right)$ to closed half planes.

\section{REFERENCES}

1. I. Colojoara and C. Foiaş, Generalized spectral operators, Gordon and Breach, London, 1968.

2. N. Dunford, A survey of the theory of spectral operators, Bull. Amer. Math. Soc. 64 (1958), 217-274.

3. N. Dunford and J. T. Schwartz, Linear operators. III: Spectral operators, Interscience, New York, 1971.

4. C. Foiaş, Some applications of spectral sets. I. Harmonic-spectral measure, Acad. R. P. Roumine. Stud. Cerc. Mat. 10 (1959), 365-401; Amer. Math. Soc. Transl. (2) 61 (1967), 25-62.

5. P. R. Halmos, A Hilbert space problem book, Van Nostrand, Princeton, NJ, 1967.

6. M. Radjabalipour, On decomposition of operators, Michigan Math. J. 21 (1974), 265-275.

7. __ Hyponormal operators and Dunford's condition (B), Math. Ann. 272 (1985), 567-575.

8. H. Radjavi and P. Rosenthal, Invariant subspaces, Springer-Verlag, Berlin, Heidelberg, and New York, 1973.

9. J. G. Stampfli, A local spectral theory for operators. III: Resolvents, spectral sets and similarity, Trans. Amer. Math. Soc. 168 (1972), 133-151.

10. B. L. Wadhwa, Spectral operators and the dual of Bergman shift, Proc. Internat. Conf. in Operator Theory, Delhi University, 1986 (to appear).

Department of Mathematics, University of Kerman, Kerman, Iran 